\title{
Differentiation of Adult Stem Cells into Smooth Muscle for Vascular Tissue Engineering
}

\author{
Lisa J. Harris, MD, Hamid Abdollahi, MD, Ping Zhang, PhD, Stephen Mcllhenny, BS, \\ Thomas Tulenko, PhD, and Paul J. DiMuzio, MD \\ Department of Surgery, Thomas Jefferson University, Philadelphia, Pennsylvania
}

\begin{abstract}
Background-Herein we evaluate the potential of adipose-derived stem cells (ASC) to differentiate into smooth muscle cells (SMC) and their potential for use in a tissue-engineered vascular graft.

Materials and Methods-We isolated ASC $(\mathrm{CD} 13+29+90+)$ from the peri-umbilical adipose tissue of patients undergoing vascular surgery, and cultured them in media containing angiotensin II (AngII), sphingosylphosphorylcholine (SPC) or transforming growth factor-beta 1 (TGF 1 ) for up to three weeks. SMC differentiation was assessed by: 1) expression of early (calponin, caldesmon) and late (myosin heavy chain, MHC) SMC markers by RT-PCR, qPCR and Western blot, and 2) contraction upon plating on collagen gel. Differentiated ASCs were seeded onto a vascular graft (decellularized saphenous vein) within a bioreactor, and cell attachment was determined using confocal microscopy.
\end{abstract}

Results-Prior to differentiation, ASC expressed low levels of all three molecular markers. After culture in each differentiating medium, the extent of up-regulation of calponin, caldesmon and MHC was variable across all cell lines. After seeding onto collagen gel, ASC differentiated in SPC and TGF $\beta 1$ exhibit contractile properties, similar to smooth muscle cell controls. Differentiated stem cells adhered and proliferated on the vascular graft.

Conclusion-These data suggest that human adipose-derived stem cells: 1) exhibit variable expression of SMC molecular markers after differentiation, 2) exhibit a contractile phenotype after differentiation with SPC and TGF $\beta 1$, and 3) proliferate on a vascular graft scaffold. Thus, ASCs are potentially useful in the construction of autologous arteries.

\section{Keywords}

adult stem cells; adipose tissue; smooth muscle cells; vascular tissue engineering

\section{Introduction}

Autologous vascular tissue remains the gold standard conduit for small-diameter $(<6 \mathrm{~mm})$ vascular bypass. Many patients afflicted with peripheral arterial disease (PAD) lack suitable autologous tissue needed for bypass due to systemic disease or prior vein harvest.1 The current alternative for these patients is bypass using a prosthetic graft or cryopreserved

Correspondence: Paul J. DiMuzio, MD, 111 South $11^{\text {th }}$ Street, Suite G6350, Philadelphia, PA 19107, 215-955-8304 (phone), 215-923-0835 (fax), paul.dimuzio@jefferson.edu.

Publisher's Disclaimer: This is a PDF file of an unedited manuscript that has been accepted for publication. As a service to our customers we are providing this early version of the manuscript. The manuscript will undergo copyediting, typesetting, and review of the resulting proof before it is published in its final citable form. Please note that during the production process errors may be discovered which could affect the content, and all legal disclaimers that apply to the journal pertain. 
allograft, both of which produce inferior results.2,3 As a result of this problem, tissue engineering strategies have emerged in an attempt to create a more suitable alternative conduit. The ultimate goal is to create a graft composed of endothelial cells (EC) and smooth muscle cells (SMC) with structure and function similar to native vessels.

Many tissue engineering strategies for creating a bypass graft have used adult stem cells harvested from bone marrow or blood. $4^{-} 8$ While both of these stem cell populations have shown potential to differentiate into mature cell lineages, cell harvest can be difficult and availability of these cells is limited by advanced patient age and the presence of comorbidities.9 $5^{-} 10$ Adipose tissue has been shown to be an abundant source of stem cells that are easily obtainable. $9,11,12$ Prior work in our laboratory has shown that adiposederived stem cells (ASC) are able to differentiate into an endothelial cell lineage that can be seeded onto a bypass graft scaffold.9'13 One next step toward the production of a tissue engineered bypass conduit is to focus on ASC differentiation toward a SMC lineage.

Smooth muscle cells are defined by both molecular markers and function. There are five molecular markers commonly used in the literature to assess for SMCs. These markers are alpha smooth muscle actin (ASMA), SM22, calponin, caldesmon, and myosin heavy chain (MHC). Alpha smooth muscle actin and SM22 are early markers of developing smooth muscle. They are not specific to a smooth muscle cell lineage and have been shown to be transcribed in pluripotent stem cells. Calponin, caldesmon and MHC are intermediate to late markers of SMC differentiation and are more specific to a SMC lineage. Smooth muscle cell function includes contraction and production of extracellular matrix.

SMC differentiation has been described in the literature using many different adult stem cell sources including bone marrow, adipose and neural crest.14-18 The majority of these studies have used soluble growth factors to induce differentiation. Factors such as transforming growth factor $\beta-1$ (TGF $\beta 1) 14^{-1} 16,18$, transforming growth factor $\beta-3$ (TGF $\left.\beta 3\right) 17$, angiotensin II (ANG)18, sphingosylphosphorylcholine (SPC)17, ascorbic acid16, and platelet-derived growth factor BB15, 16 have been used to induce varying degrees of SMC differentiation. Culture conditions differ across the studies, including the concentration of the growth factor and the type of media used. Different stock media are used with varying concentrations of glucose and fetal bovine serum (FBS). Occasionally the media is enhanced with other supplements in addition to the differentiating agent being evaluated. Additionally, the various studies use different molecular markers to assess differentiation. This lack of consistency across studies makes it difficult to compare and interpret results.

The purpose of this study is to investigate the influence of certain soluble factors on SMC differentiation of ASC. By using a single stem cell type and consistent, standardized culture conditions, our goal is to compare the potential of the soluble factors ANG, SPC and TGF $\beta 1$ to drive ASC toward a SMC phenotype. The SMC phenotype will be assessed on a molecular level by the expression of calponin, caldemon, and MHC, and functionally based on contractility. We will also explore the potential for use of these differentiated ASC in the creation of a tissue-engineered vascular bypass graft.

\section{Materials and Methods}

The Thomas Jefferson University Institutional Review Board (IRB) approved of all human studies. All patients gave informed consent prior to tissue donation.

\section{ASC Isolation}

Human adipose tissue was collected from the peri-umbilical region of patients undergoing elective vascular surgical procedures via liposuction aspiration (Table 1). The adipose tissue 
was digested in Collagenase I (Worthington, Biochemical Corp, Lakewood, NJ; 4 mg/gm tissue) for $1 \mathrm{~h}$ at $37^{\circ} \mathrm{C}$. Next, it was centrifuged at $1500 \times \mathrm{g}$ to separate the cellular component. After discarding the supernatant, the resultant stromal-vascular pellet was suspended in non-differentiating media (Media-199 (Mediatech, Herndon, VA) supplemented with $13 \%$ fetal bovine serum (Gemini Bio-Products, West Sacramento, CA), antibiotic-antimycotic solution (Mediatech; $12 \mathrm{~mL} / \mathrm{L}$ ) and heparin $(7.5 \mathrm{U} / \mathrm{mL}$ ). Finally the stromal vascular fraction, containing our ASC population $(\mathrm{CD} 13+29+90+)$, was plated on gelatin-coated culture flasks $\left(1 \times 10^{6}\right.$ cells per flask) at $37^{\circ} \mathrm{C}$ in $5 \% \mathrm{CO}_{2}$. Previous analysis has shown that greater than $98 \%$ of the cell population are CD13+29+90+, and that ASC cultures were morphologically homogeneous. 13

\section{Differentiation of ASC}

ASC were differentiated toward a smooth muscle cell lineage via exposure to soluble factors. Differentiating media consisted of Media-199 (Mediatech, Herndon, VA), 13\% fetal bovine serum (Gemini Bio-Products, West Sacramento, CA), antibiotics (Mediatech; $12 \mathrm{~mL} /$ $\mathrm{L})$, heparin $(7.5 \mathrm{U} / \mathrm{mL})$ and one of the following supplements: 1) $1 \mu \mathrm{M}$ angiotensin II (Sigma-Aldrich, St. Louis, MO), 2) $2 \mu \mathrm{M}$ sphingosylphosphorylcholine (Matreya, LLC; Pleasant Gap, PA), or 3) 2 ng/ml TGF- $\beta 1$ (R\&D Systems, Inc., Minneapolis, MN).17,18 ASC were cultured in differentiating media for up to three weeks. Media was changed twice weekly throughout the culture period.

\section{Cell Proliferation Assay and Doubling Time}

Cell counts were performed every 24 hours for seven days using a Coulter counter (Beckman Coulter, Inc, Fullerton CA) for ASC ( $n=3)$ grown in each of our four culture medias: non-differentiating media (no supplements added), ANG, SPC, and TGF $\beta 1$. Starting cell counts were $2 \times 10^{4}$ for each cell population. Doubling time $\left(\mathrm{T}_{\mathrm{d}}\right)$ was calculated using the following formula: $T_{d}=\left(t_{1}-t_{0}\right) \log 2 / \log \left(N_{1} / N_{0}\right)$ where $t_{1}$ is the time of the second cell count, $\mathrm{t}_{0}$ is the time of the first cell count, $\mathrm{N}_{1}$ is the number of cells in the second cell count, and $\mathrm{N}_{0}$ is the number of cells in the first cell count.

\section{Evaluation of Differentiation}

Differentiation of ASC was evaluated by 1) expression of SMC markers, and 2) assessment of contractile function. Human arterial smooth muscle cells (American Type Culture Collection, Manassas, VA) were used as positive control and undifferentiated ASC were used as negative control.

Expression of SMC Specific Markers: RT-PCR-Expression of smooth muscle cell markers were identified by isolating total RNA from differentiated cells after one and two weeks in culture. Total RNA was extracted through RNeasy mini columns (QIAGEN, Valencia, CA). RNA concentration was obtained via spectrophotometer analysis. Reverse transcription was then performed by using the Promega Reverse Transcription System (Promega, Madison, WI). One microgram of total RNA was used in each reaction. The following primer pairs (Operon, Huntsville, AL) were used: Calponin (Forward 5'ATGTCCTCTGCTCACTTCA3'; Reverse 5'TTTCCGCTCCTGCTTCTCT3'), Caldesmon (Forward 5'AGATTGAAAGGCGAAGAGCA3'; Reverse 5'TTCAAGCCAGCAGTTTCCTT3'), MHC (Forward 5'GGACGACCTGGTTGTTGATT3'; Reverse 5'GTAGCTGCTTGATGGCTTCC3'). Gel electrophoresis was then performed on a $2 \%$ agarose gel treated with ethidium bromide and visualized using a UV light box. 
Expression of SMC Specific Markers: Quantitative PCR-Total RNA was isolated from differentiated cells after one and two weeks in culture using RNeasy Mini Kit (QIAGEN, Valencia, CA) according to manufacturer's protocol. TaqMan Gene Expression Assays targeting the smooth muscle cell markers calponin, caldesmon and MHC were obtained (Hs00154543_m1 CNN1, Hs00263989_m1 CALD1, Hs00224610_m1 MYH11; Applied Biosystems, Foster City, CA). Gene transcripts were measured by TaqMan realtime reverse transcription PCR with the 7500 Fast Real-Time PCR system (Applied Biosystems, Foster City, CA). Human GAPDH was used as internal control for standardization.

Expression of Cell Specific Markers: Western Blot-Total protein was extracted from differentiated cells using a sodium dodecyl sulfate derived buffer and quantified using BioRad Protein Assay Kit (BioRad, Hercules, CA). Proteins were separated on a 4-12\% graded tris-glycine gel (Invitrogen, Carlsbad, CA) and transferred to a polyvinylidene difluoride membrane (Invitrogen, Carlsbad, CA). Membranes were blocked with Odyssey Blocking Buffer (LI-COR Biosciences, Lincoln, NE) then incubated overnight with primary antibody in Odyssey Blocking Buffer/0.1\% Tween 20. The following primary antibodies were used: anti-calponin, anti-caldesmon, and anti-MHC (Santa Cruz Biotechnology, Inc., Santa Cruz, CA). Bound primary antibody was labeled with horseradish peroxidase (HRP)conjugated goat anti-mouse or goat anti-rabbit (Santa Cruz Biotechnology, Inc., Santa Cruz, CA). Signals were detected using the Odyssey ${ }^{\circledR}$ Imaging System (LI-COR Biosciences, Lincoln, NE).

Assessment of Function: Collagen gel lattice contraction assay-In order to measure contractility, a collagen gel lattice contraction assay was performed.19

Differentiated ASC were trypsinized from monolayer culture, counted and resuspended in Media-199 at a density of $1 \times 10^{6}$ cells $/ \mathrm{ml}$. The collagen lattice was prepared by combining $270 \mathrm{ul}$ of collagen solution (PurCol, Advanced BioMatrix), $15 \mathrm{ul}$ sodium bicarbonate, and $30 \mathrm{ul}$ of 10x Media-199. Two hundred microliters of the cell solution were then added to the collagen lattice mixture. The cell-collagen mixture was pipetted into 24 well culture plates and incubated for 90 minutes at $37^{\circ} \mathrm{C}$ to polymerize the collagen cell lattices. After 90 minutes the collagen lattices were mechanically released from the culture dish by pipetting either serum-free medium alone or serum-free medium containing $60 \mathrm{mM} \mathrm{KCl}$ at the latticedish interface. Serum-free medium alone was used to determine basal contractile tone of the cells, while the addition of $\mathrm{KCl}$ was to assess a stimulated contractile response as a result of membrane depolarization. A digital camera at a fixed distance was used to take pictures of the lattices at specific time points after release. The area of the gel lattice was calculated using NIH Image $\mathbf{J}$ software. The relative amount of contraction was obtained using the following formula: relative gel area $=$ gel surface area at specific time point/initial gel surface area. Percent increase in contraction was calculated using the following formula: relative gel area of cells in serum-free medium containing $\mathrm{KCl}$ - relative gel area of cells in serum-free medium alone.

\section{Attachment to Vascular Graft Scaffold}

Human greater saphenous vein (Regeneration Technologies Inc., Birmingham, AL) was decellularized by placing the specimen in a $0.075 \%$ SDS (Sigma Chemical Company, St. Louis, MO) solution in a $37^{\circ} \mathrm{C}$ shaking water bath for 15 hours. The vein is then washed with agitation in phosphate-buffered saline five times. Differentiated ASCs were trypsinized from monolayer culture, counted and resuspended in DMEM at a concentration of $1 \times 10^{7}$ cells $/ \mathrm{ml}$. Fifty microliters of cell suspension was applied to the abluminal surface of the decellularized vein and allowed to incubate at $37^{\circ} \mathrm{C}$ for one hour. The vein was flipped over and an additional $50 \mu \mathrm{l}$ of cell suspension was applied to the other side of the vein and 
allowed to incubate for one hour. At this time, the seeded decellularized vein was placed onto a bioreactor system (Tissue Growth Technologies, Minnetonka, MN) and DMEM media was circulated around the abluminal surface of the vein. After 24 hours, the vein was harvested from the bioreactor system and the abluminal surface was stained with $2 \mathrm{ml}$ of Cell Tracker Green (Invitrogen, Carlsbad, California). Cell attachment was assessed using confocal microscopy at $40 \times$ magnification.

\section{Statistical Analysis}

All values are expressed as mean \pm standard deviation. Statistical analysis was performed on quantified data (qPCR and contraction assay) using a two-tailed Student's t-test. A p-value less than 0.05 was considered significant.

\section{Results}

\section{Proliferation Characteristics}

Undifferentiated ASC showed similar proliferation when compared to ASC grown in media supplemented with ANG, SPC or TGF 31 (Figure 1). The doubling times for each of the four culture conditions between 0 and 7 days were: undifferentiated ASC $2.9 \pm 0.2$, ANG $3.0 \pm$ 0.2 , SPC $2.8 \pm 0.2$, and TGF $\beta 12.7 \pm 0.1$. There were no significant differences between these four groups with regard to doubling times.

\section{Effect of ANG, SPC, and TGF $\beta 1$ on the Expression of SMC Markers}

Prior to culture with the three differentiating agents, ASC did not express calponin or MHC, however expression of caldesmon was seen in two of four patient cell lines as evidenced by RT-PCR (Figure 2). After one week in culture, expression of calponin was seen after differentiation with all three agents in one cell line only. All other cell lines did not express calponin. Expression of caldesmon was variable among cell lines. Two cell lines did not show up-regulation of caldesmon, one showed up-regulation with all three agents, and another showed up-regulation with ANG only. No MHC expression was seen with any cell lines at one week.

After two weeks of differentiation, up-regulation of calponin expression was seen with TGF $\beta 1$ in one cell line only by RT-PCR (Figure 3). All others did not show up-regulation of calponin. At two weeks, expression of caldesmon was slightly variable with only half of the cell lines showing up-regulation of caldesmon. Expression of MHC was seen with TGF $\beta 1$ in three cell lines and with SPC in another cell line at two weeks, while two cell lines did not show any MHC expression.

When SMC marker expression was quantified using quantitative PCR (Figure 4), results remained variable across the different cell lines. Up-regulation of calponin was seen with TGF $\beta 1$. No up-regulation of caldesmon was achieved by any of the stimulating agents. Some up-regulation of MHC was seen with ANG and SPC. However, no consistent pattern of expression was observed across all cell lines for any of the SMC markers.

Western blot analysis after three weeks in culture (Figure 5) revealed up-regulation of calponin protein with all three agents in two of three cell lines. A third cell line showed upregulation of calponin protein with TGF $\beta 1$ only. No up-regulation of caldesmon protein was observed with any of the agents, although caldesmon protein was expressed at baseline and with all agents. No expression of MHC protein was seen. 


\section{Assessment of Function: Collagen Gel Lattice Contraction Assay}

Unseeded collagen gels maintain their surface area over time, therefore any change in surface area is due to contraction from cells seeded within the gel (Figure 6). Basal contractile tone is exhibited by undifferentiated ASC and stem cells differentiated with each of the three agents. The amount of basal tone is less than the amount exhibited by the positive control (human arterial SMCs). The addition of $\mathrm{KCl}$ results in a significant increase in contraction with ASC differentiated with SPC and TGF $\beta 1$ compared to undifferentiated ASC, similar to the positive control. No increase in contraction was seen with ASC differentiated in ANG.

\section{Attachment to Vascular Graft Scaffold}

ASC differentiated in either ANG, SPC or TGF $\beta 1$ attach to abluminal surface of decellularized human greater saphenous vein after 24 hours in bioreactor system as viewed by confocal microscopy (Figure 7). Cells remain attached to vein and viable as long as one week in the bioreactor system.

\section{Discussion}

In the present study we evaluated the effect of three growth factors (ANG, SPC, and TGFß1) on differentiation of human adipose-derived stem cells toward a SMC lineage and the potential use of the differentiated cells in the creation of a tissue engineered vascular bypass graft. We observed considerable variation in expression of the SMC markers calponin, caldesmon, and $\mathrm{MHC}$ at both the gene and protein levels with all three growth factors across different cell lines. Assessment of functionality revealed that ASC differentiated in SPC and TGF $\beta 1$ showed contraction in response to stimulation by $\mathrm{KCl}$, a characteristic of differentiated SMCs. Stem cells differentiated in either ANG, SPC or TGFB were able to be seeded successfully onto a decellularized vascular graft.

To date, only two other laboratories have published data on the differentiation of human ASCs into SMCs.17,18,20 Rodriquez et al used a smooth muscle inductive medium (Medium MCDB $131+1 \%$ fetal bovine serum +100 units/ml heparin) to achieve differentiation.20 They demonstrated up-regulation of the markers alpha smooth muscle actin (ASMA), calponin, caldesmon, SM22, smoothelin and MHC by RT-PCR and upregulation of ASMA and MHC by Western blot analysis. In our study, cells were cultured in media comprised of Medium 199, 10\% fetal bovine serum, penicillin/streptomycin and the addition of either ANG, SPC, TGF $\beta 1$ or no growth factors. Due to difference in culture media, we are not able to support or refute their claim. However, we did not achieve the same up-regulation of markers calponin, caldesmon and $\mathrm{MHC}$ reported in this study.

In contrast, a second set of collaborators reported that ANG, SPC, TGF $\beta 1$, and TGF $\beta 3$ added to culture media comprised of $\alpha$-minimum essential medium with $10 \%$ fetal bovine serum and penicillin/streptomycin, all induced SMC differentiation by human ASCs.17,18 Jeon et al reported up-regulation of the markers ASMA, calponin, and SM22 by SPC and TGF $\beta 3$ as evidenced by RT-PCR and qPCR, and up-regulation of ASMA by SPC, TGF $\beta 1$ and TGF $\beta 3$ on Western blot analysis.17 Both ASMA and SM22 are early markers of developing smooth muscle. They are not specific to a SMC lineage and have been shown to be spontaneously expressed in pluripotent stem cells. We found both ASMA and SM22 to be transcribed in our native ASC population on RT-PCR analysis (data not shown), therefore, we did not use these markers in our evaluation of differentiation. Our RT-PCR and qPCR analysis did not show consistent up-regulation of calponin by SPC across all cell lines. Therefore, we cannot support their claim that SPC induces SMC differentiation in human ASCs. 
In a second publication, this same lab reported up-regulation of ASMA, calponin, caldesmon, and MHC by both ANG and TGF $\beta 1$ on Western blot analysis. 18 Our Western blot analysis of differentiation demonstrated up-regulation of calponin by ANG in two of three cell lines and by TGF $\beta 1$ in all three cell lines. We did not observe any up-regulation of caldesmon or MHC by either ANG or TGF $\beta 1$. Based on these results, we agree that ANG and TGF $\beta 1$ drive ASCs toward a SMC lineage based on expression of calponin, but cannot say that it induces a fully differentiated state due to lack of MHC expression. The present study showed that neither ANG, SPC or TGF $\beta 1$ were able to induce adipose-derived stem cells to a fully differentiated SMC phenotype based on the expression of molecular markers, however, all three agents do drive SMC differentiation in adipose-derived stem cells to some degree.

In addition to molecular markers, we also studied our differentiated stem cells to see if they acquired the contractile function of smooth muscle. We found that ASCs differentiated in SPC and TGF $\beta 1$ expressed basal contractile tone and demonstrated a significant increase in contraction in response to stimulation with $\mathrm{KCl}$, both characteristics of smooth muscle. Contractile response to $\mathrm{KCl}$ was not observed in ASC differentiated in ANG. This contractile function of differentiated ASC has important implications for vascular tissue engineering. Contractility adds functional properties to a vascular graft that are similar to native arteries. It provides added strength to the graft, thereby increasing the ability of the graft to withstand physiologic stresses. 21 This results in a graft with sufficient strength not to burst with changes in blood pressure. The addition of contractile function creates a vasoreactive graft that is able to alter its diameter and compliance in response to hemodynamic forces and chemical stimuli. Thus, the contractile function of differentiated ASC is important in creating a functional vascular conduit that possesses the mechanical properties of a native blood vessel.

Smooth muscle cells are important in the creation of tissue-engineered blood vessels. They provide increased strength to vessels when circumferentially oriented creating a graft that can withstand cyclic loading and is able to handle changes in arterial blood pressure without rupture.22,23 The contractile function of SMCs creates a graft that is vasoreactive to external stimuli. This allows propagation of pulsatile flow and physiologic adjustments in flow based on cardiovascular demand. The synthetic function of SMCs results in production of extracellular matrix (ECM) comprised of collagen, elastin and proteoglycans which allows for elasticity and improved compliance of the graft. For these reasons, SMCs are an integral part in constructing a bypass graft. Several tissue-engineered grafts using SMCs have been constructed. Ratcliffe created a graft consisting of a polyurethane scaffold seeded with SMCs and ECs cultured under fluid flow.24 When implanted into canine carotid arteries, the grafts remained patent and free from intimal hyperplasia for four weeks. ShumTim et al seeded polyglycolic acid (PGA)-polyhydroxyalkanoate (PHA) co-polymer scaffolds with ovine carotid ECs and SMCs.25 When implanted in the infrarenal aortas of lambs, the grafts remained patent for five months and over time showed collagen content and mechanical stress curves similar to those of native aorta. Niklason et al seeded bovine aortic SMCs and ECs onto a PGA scaffold placed around a distensible silicon tube in a biomimetic system. 26 When the silicon tube was removed, the resultant graft had collagen content and wall thickness comparable to native arteries and a burst strength greater than that reported for native human greater saphenous veins. Niklason et al also described the use of bone marrow-derived stem cells in the creation of a tissue-engineered graft.27 They seeded a PGA scaffold with stem cells in a biomimetic system for eight weeks under conditions for stem cell proliferation and differentiation. The result was a graft containing stem cells differentiated toward a SMC lineage and increased collagen and extracellular matrix content. In this study, we have shown that adipose-derived stem cells differentiated toward a SMC lineage by ANG, SPC or TGF $\beta 1$ are able to attach to a vascular graft scaffold 
(decellularized saphenous vein). Thus, adipose-derived stem cells have the potential for use in the creation of tissue engineered blood vessels.

The main weakness of our current study is the variability of SMC marker expression experienced across different cell lines. Consistent expression of various SMC markers has been reported using stem cells from a variety of sources. One explanation for our variability in expression could be due to loss of differentiation potential by the adipose-derived stem cells that is not seen in other cell lines. As discussed above, others have reported consistent expression of SMC markers using adipose-derived stem cells, however this was not our experience.17,18,20 It is also possible that the variability in expression is due to differences between our study and others in the patient population from which our adipose samples were obtained. Our samples were harvested from older patients (mean age 65 years), the majority of who had one or more comorbid conditions (Table 1). The other studies using ASC for SMC differentiation do not describe their patient population.17,18,20 Perhaps these patient populations consist of younger patients, fewer comorbidities or other differences that may have resulted in less variability. Another reason for variable SMC marker expression could be from poor stimulation of differentiation. This seems less likely since the agents used have been previously reported to induce differentiation at similar concentrations.

In summary, these data suggest that human adipose-derived stem cells: 1) exhibit variable expression of SMC molecular markers after differentiation, 2) exhibit a contractile phenotype after differentiation with SPC and TGF 31 , and 3) can attach to a vascular graft scaffold. Thus, adipose-derived stem cells can be driven toward a SMC lineage and have potential for use in the construction of autologous arteries.

\section{Acknowledgments}

This work was supported by the following grants: NIH K08 HL076300-01 (P.J.D., T.N.T.) and the American Vascular Association (P.J.D.). We thank Charles J. Yeo, M.D., Samuel D. Gross Professor and Chairman of the Department of Surgery, for critical review of the manuscript.

\section{References}

1. Veith FL, Moss CM, Sprayregen S, Montefusco C. Pre-operative saphenous venography in arterial reconstructive surgery of the lower extremity. Surgery. 1979; 85:253. [PubMed: 424995]

2. Bergan JJ, Veith FJ, Bernhard VM, et al. Randomization of autogenous vein and polytetrafluoroethylene grafts in femoral-distal reconstruction. Surgery. 1982; 92:921. [PubMed: 6755789]

3. Veith FJ, Gupta SK, Ascer E, et al. Six year prospective multicenter randomized comparison of autologous saphenous vein and expanded polytetrafluoroethylene grafts in infrainguinal arterial reconstructions. J Vasc Surg. 1986; 3:104. [PubMed: 3510323]

4. Riha GM, Lin PH, Lumsden AB, et al. Application of stem cells for vascular tissue engineering. Tissue Eng. 2005; 11:1535. [PubMed: 16259608]

5. Barrilleaux B, Phinney DG, Prockop Darwin, O'Connor KC. Review: Ex vivo engineering of living tissues with adult stem cells. Tissue Eng. 2006; 12:1. [PubMed: 16499437]

6. Levenberg S. Engineering blood vessels from stem cells: recent advances and application. Curr Opin Biotechnol. 2005; 16:516. [PubMed: 16144762]

7. Isenberg BC, Williams C, Tranquillo Rt. Small-diameter artificial arteries engineered in vitro. Circ Res. 2006; 98:25. [PubMed: 16397155]

8. Sales KM, Salacinski HJ, Alobaid N, et al. Advancing vascular tissue engineering: the role of stem cell technology. Trends Biotechnol. 2005; 9:461. [PubMed: 15979750]

9. DiMuzio P, Tulenko T. Tissue engineering applications to vascular bypass graft development: The use of adipose-derived stem cells. J Vasc Surg. 2007; 45:99A. 
10. Dzau VJ, Gnecchi M, Pachori AS, et al. Therapeutic potential of endothelial progenitor cells in cardiovascular diseases. Hypertension. 2005; 46:7. [PubMed: 15956118]

11. Katz AJ, Tholpady A, Tholpady SS, et al. Cell surface and transcriptional characterization of human adipose-derived adherent stromal (hADAS) cells. Stem Cells. 2005; 23:412. [PubMed: 15749936]

12. Mitchell JB, McIntosh K, Zvonic S, et al. Immunophenotype of human adipose-derived stem cells: temporal changes in stromal-associated and stem cell-associated markers. Stem Cells. 2006; 24:376. [PubMed: 16322640]

13. Fischer LJ, McIlhenny S, Tulenko T, et al. Endothelial differentiation of adipose-derived stem cells: Effects of endothelial growth supplement and shear force. J Surg Res. 2009; 152:157. [PubMed: 19883577]

14. Gong Z, Calkins G, Chen E, et al. Influence of culture medium of smooth muscle cell differentiation from human bone marrow-derived mesenchymal stem cells. Tissue Engineering. 2009; 15:319. [PubMed: 19115826]

15. Chen S, Lechleider R. Transforming growth factor- $\beta$-induced differentiation of smooth muscle from a neural crest stem cell line. Circ Res. 2004; 94:1195. [PubMed: 15059931]

16. Narita Y, Yamawaki A, Kagami H, et al. Effects of transforming growth factor-beta 1 and ascorbic acid on differentiation of human bone-marrow-derived mesenchymal stem cells into smooth muscle cell lineage. Cell Tissue Res. 2008; 333:449. [PubMed: 18607632]

17. Jeon ES, Moon HJ, Lee MJ, et al. Sphingosylphosphorylcholine induces differentiation of human mesenchymal stem cells into smooth-muscle-like cells through a TGF- $\beta$-dependent mechanism. J Cell Sci. 2006; 119:4994. [PubMed: 17105765]

18. Kim YM, Jeon ES, Kim MR, et al. Angiotensin II-induced differentiation of adipose tissue-derived mesenchymal stem cells to smooth muscle-like cells. Int J Biochem Cell Biol. 2008; 40:2482. [PubMed: 18571460]

19. Woodman L, Siddiqui S, Cruse G, et al. Mast cells promote airway smooth muscle cell differentiation via autocrine up-regulation of TGF- $\beta 1$. J Immunol. 2008; 181:5001. [PubMed: 18802103]

20. Rodriguez LV, Alfonso Z, Zhang R, et al. Clonogenic multipotent stem cells in human adipose tissue differentiate into functional smooth muscle cells. PNAS. 2006; 103:12167. [PubMed: 16880387]

21. Wu HC, Wang TW, Kang PL, et al. Coculture of endothelial and smooth muscle cells on a collagen membrane in the development of a small-diameter vascular graft. Biomaterials. 2007; 28:1385. [PubMed: 17141865]

22. Kakisis JD, Liapis CD, Breuer C, et al. Artificial blood vessel: The holy grail of peripheral vascular surgery. J Vasc Surg. 2005; 41:349. [PubMed: 15768021]

23. L'Heureux N, Paquet S, Labbe R, et al. A completely biological tissue-engineered human blood vessel. FASEB J. 1998; 12:47. [PubMed: 9438410]

24. Ratcliffe A. Tissue engineering of vascular grafts. Matrix Biology. 2000; 19:353. [PubMed: 10963996]

25. Shum-Tim D, Stock U, Hrkach J, et al. Tissue engineering of autologous aorta using a new biodegradable polymer. Ann Thoracic Surg. 1999; 68:2298.

26. Niklason LE, Gao J, Abbott WM, et al. Functional arteries grown in vitro. Science. 1999; 284:489. [PubMed: 10205057]

27. Niklason LE, Gong Z. Small-diameter human vessel wall engineered from bone marrow-derived mesenchymal stem cells (hMSCs). FASEB J. 2008; 22:1635. [PubMed: 18199698] 


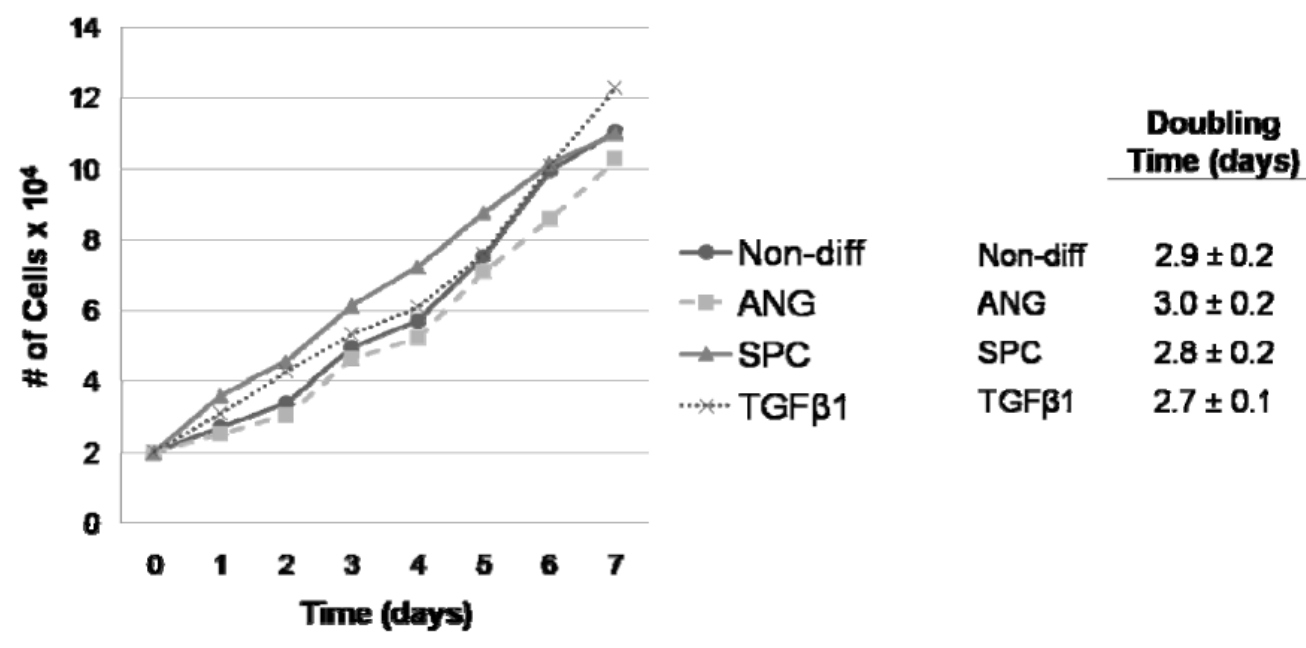

Figure 1. Proliferation of ASC and doubling times

Growth curve over 7 days showing ASC $(\mathrm{n}=3)$ cultured in non-differentiating media and with media containing growth factors ANG, SPC and TGF $\beta 1$ (error bars omitted due to juxtaposition of lines). All p-values for points on curve are not significant. Similarly, doubling times are not significantly different. 


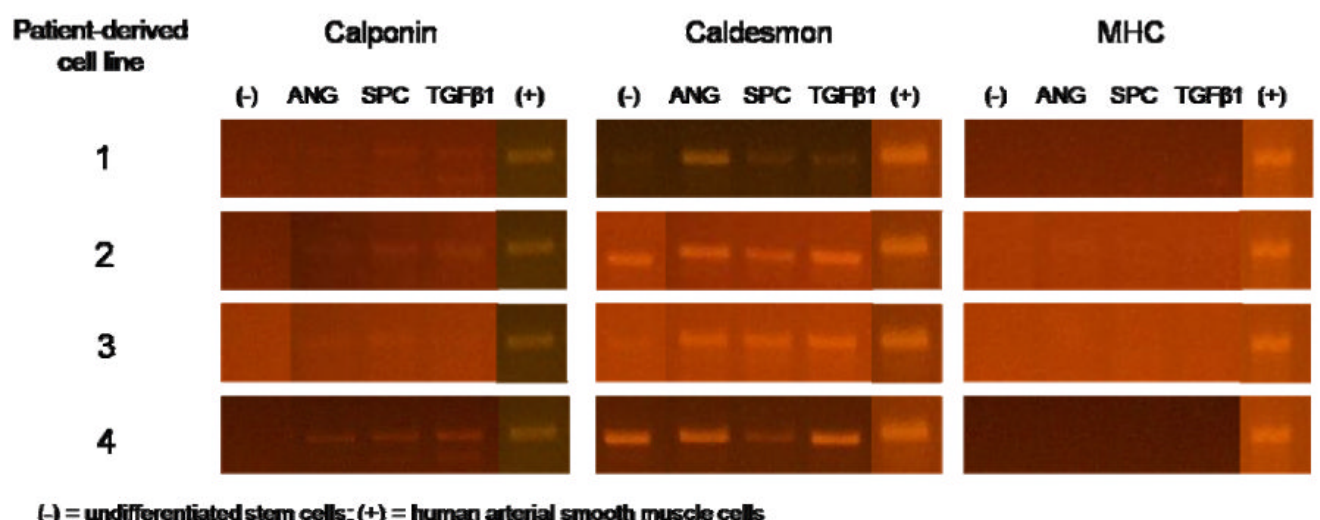

Figure 2. Expression of intermediate and late SMC markers after one week of differentiation RT-PCR for calponin, caldesmon, and MHC was performed for 4 patient cell lines after differentiation in ANG, SPC or TGF $\beta 1$ for one week. Expression of calponin was seen with all 3 differentiating agents in patient 4 only. Up-regulation of caldesmon was seen in patients 1 and 3 only. No MHC expression was seen with any cell lines at one week. 


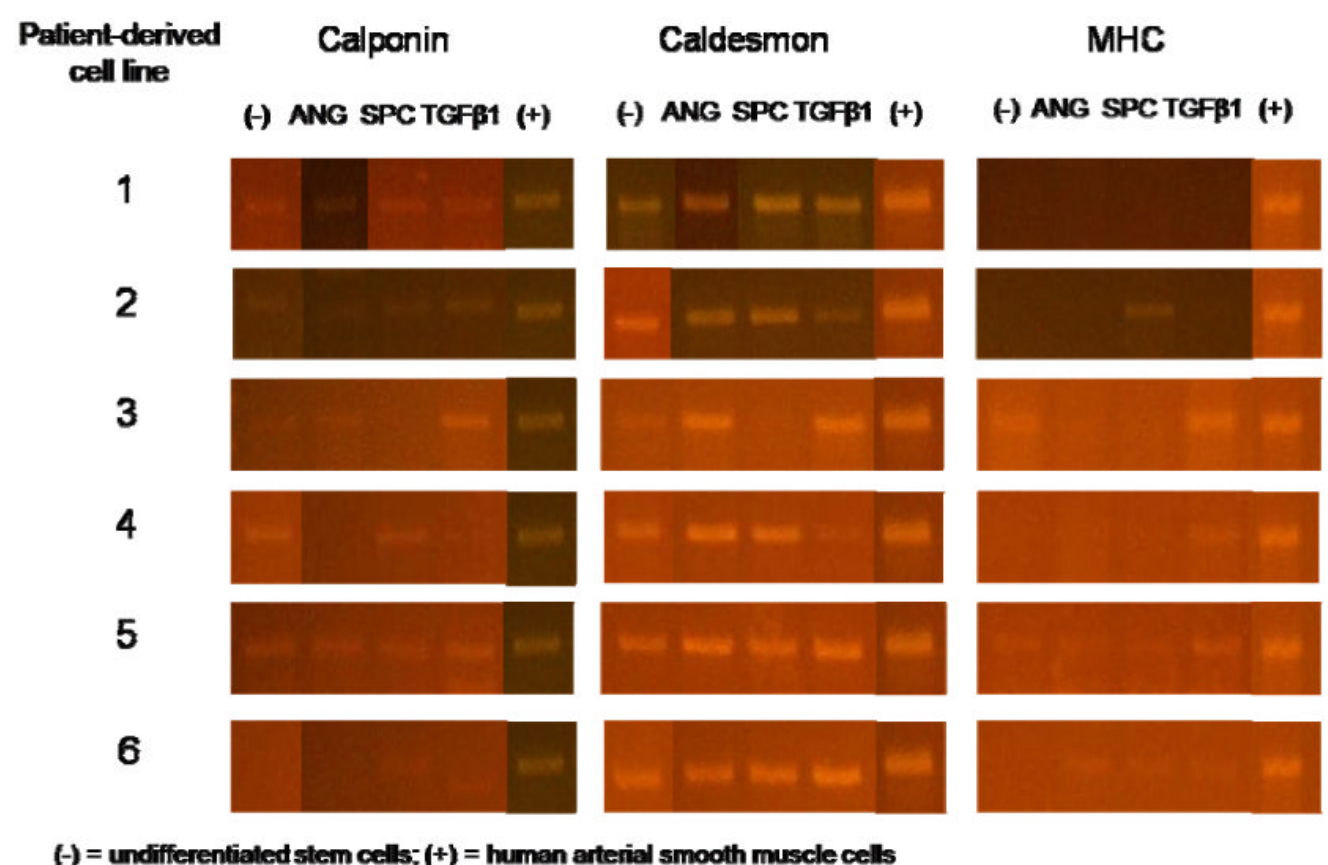

Figure 3. Expression of intermediate and late SMC markers after differentiation for two weeks RT-PCR for calponin, caldesmon, and MHC was performed in 6 patient cell lines after differentiation for two weeks. Up-regulation of calponin was seen in patient 3 only with TGF $\beta 1$. Caldesmon up-regulation was variable with 3 of the 6 patients showing upregulation. Expression of MHC was seen with SPC in patient 2 and with TGF $\beta 1$ in patients 3-5. Patients 1 and 6 did not show MHC expression. 

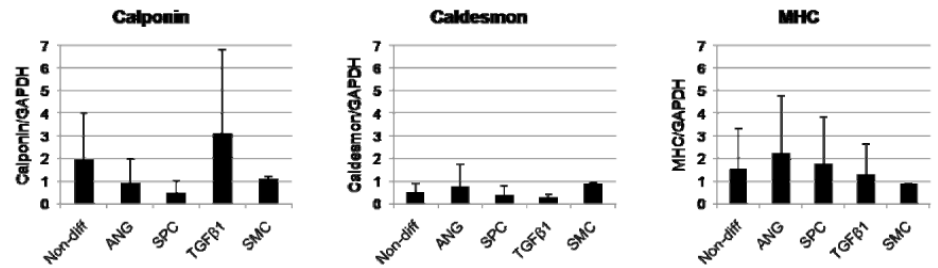

Figure 4. Quantification of SMC marker expression after two weeks of differentiation Quantitative PCR for calponin, caldesmon, and MHC after ASC differentiation $(n=3)$ for two weeks. Up-regulation of calponin was seen with TGF $\beta 1$. No up-regulation of caldesmon was seen by ANG, SPC, or TGF $\beta 1$. Up-regulation of MHC was seen with ANG and SPC. Pvalues were not statistically significant. 

Patient-derived
cell line
Figure 5. Protein expression after three weeks of differentiation Expression of calponin, caldesmon, and MHC protein by Western blot analysis after differentiation in ANG, SPC, or TGF $\beta 1$ for three weeks. Up-regulation of calponin was seen with TGF $\beta 1$ in cell line 7 and with all three agents in cell lines 8 and 9. No up-regulation of caldesmon was seen, however caldesmon was expressed at baseline and with all three differentiating agents. No expression of MHC was seen. 

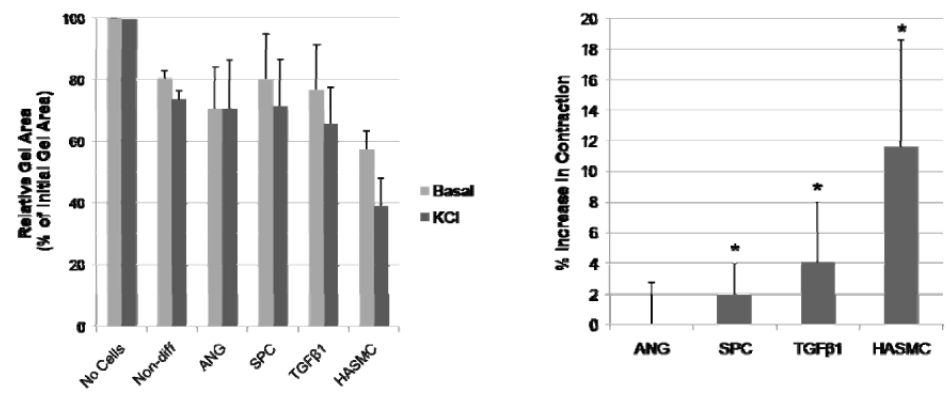

$N=4, " p<0.05$, HASMC $=$ Human anterial smooth muscle cells

Figure 6. Assessment of contraction: Collagen gel contraction assay

A. Assessment of basal contractile tone and stimulation of contraction by $\mathrm{KCl}$ using collagen gel lattice contraction assay $(n=4)$. Collagen gels without cells show no evidence of contraction. Some basal contractile tone is exhibited by non-differentiated ASC and stem cells differentiated in ANG, SPC and TGF $\beta 1$. The addition of $\mathrm{KCl}$ results in increased contraction with ASC differentiated with SPC and TGF $\beta 1$, but not ANG when compared to undifferentiated ASC. Human arterial smooth muscle cells were used as a positive control. B. Percent increase in contraction after stimulation with $\mathrm{KCl}$ normalized to undifferentiated ASC. Stem cells differentiated in SPC and TGF $\beta 1$ show significantly increased contraction $(\mathrm{p}<0.05)$ similar to human arterial SMCs. 


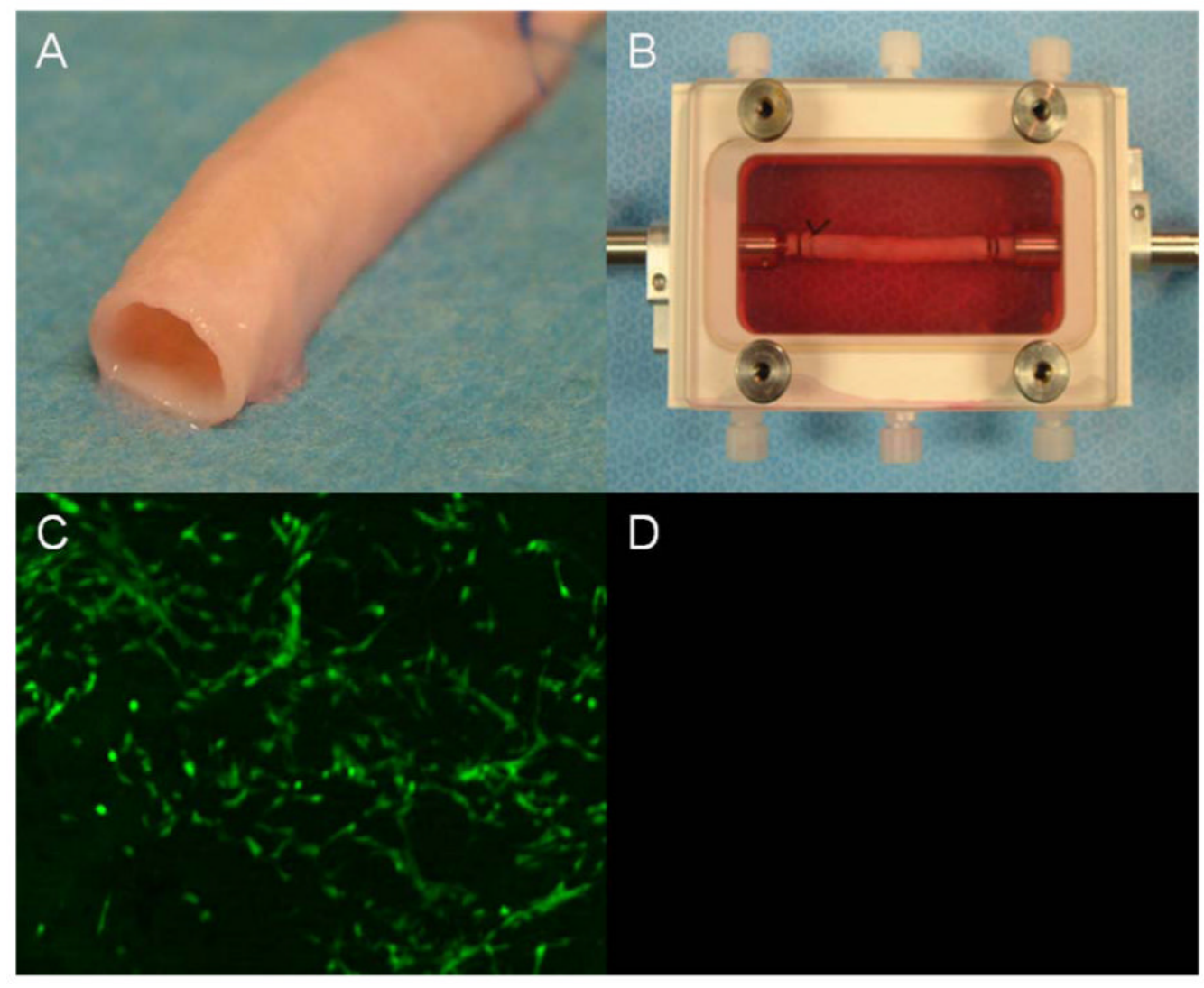

Figure 7. Attachment of differentiated ASC to vascular graft scaffold ASCs differentiated in SPC for two weeks were seeded onto vascular graft scaffold (decellularized human greater saphenous vein) (A) and cultured in bioreactor system (B) for 24 hours. Cell attachment as viewed by confocal microscopy at 40× magnification (C). Similar results were obtained with cells differentiated in ANG and TGF $\beta 1$. Vascular graft scaffold as viewed by confocal microscopy prior to seeding (D). 


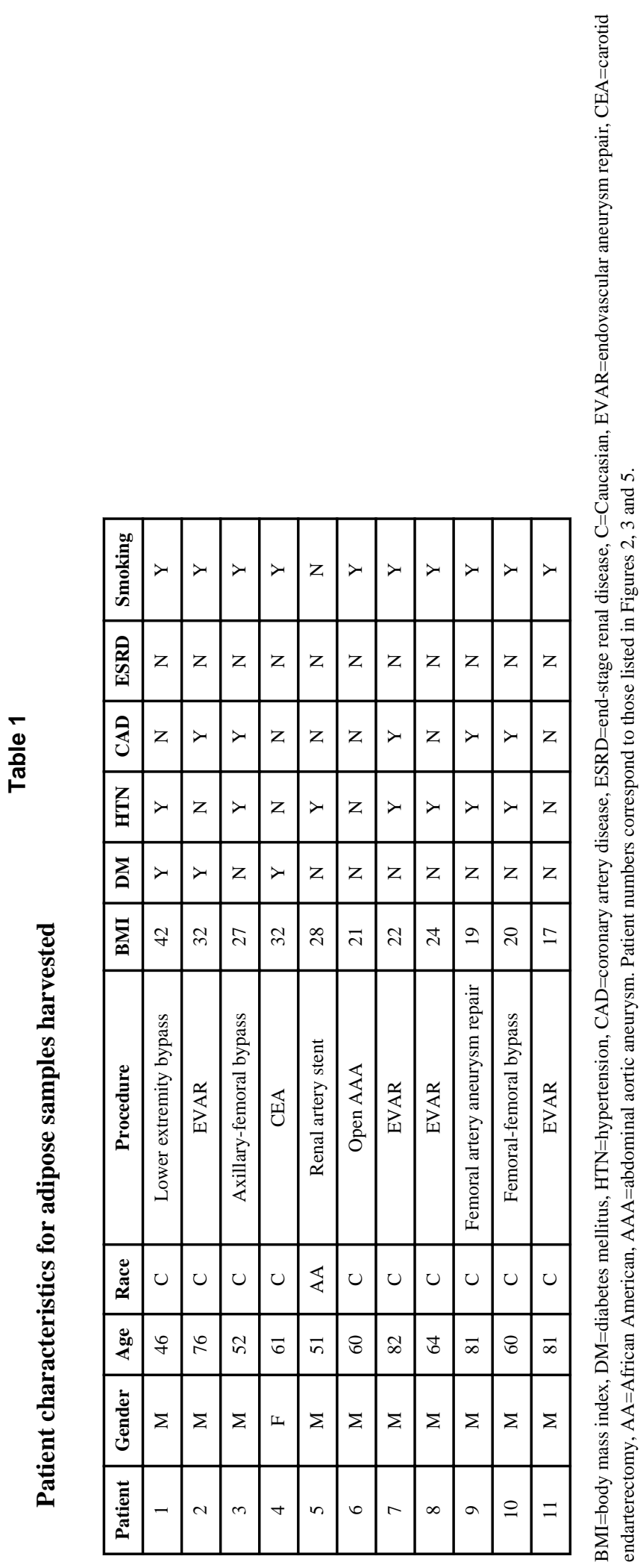

J Surg Res. Author manuscript; available in PMC 2012 June 15. 